\title{
In Vivo Functional Effects of CYP2C9 M1L, a Novel and Common Variant in the Yup'ik Alaska Native Population ${ }^{\mathbb{S}}$
}

\author{
DLindsay M. Henderson, Scarlett E. Hopkins, Bert B. Boyer, Timothy A. Thornton, Allan E. Rettie, \\ and Kenneth E. Thummel
}

Departments of Pharmaceutics (L.M.H., K.E.T.), Biostatistics (T.A.T.), and Medicinal Chemistry (A.E.R.), University of Washington, Seattle, Washington; and Department of Obstetrics and Gynecology (S.E.H., B.B.B.), Oregon Health \& Science University, Portland, Oregon

Received October 30, 2020; accepted February 4, 2021

\section{ABSTRACT}

Alaska Native people are under-represented in genetic research but have unique gene variation that may critically impact their response to pharmacotherapy. Full resequencing of CYP2C9 in a crosssection of this population identified CYP2C9 Met1Leu (M1L), a novel, relatively common single nucleotide polymorphism hypothesized to confer CYP2C9 poor metabolizer phenotype by disrupting the start codon. $M 1 L$ is present at a minor allele frequency of $6.3 \%$ in Yup'ik Alaska Native people and thus can contribute to the risk of an adverse drug response from narrow-therapeutic-index CYP2C9 substrates such as (S)-warfarin. This study's objective was to characterize the catalytic efficiency of the Leu1 variant enzyme in vivo by evaluating the pharmacokinetic behavior of naproxen, a probe substrate for CYP2C9 activity, in genotyped Yup'ik participants. We first confirmed the selectivity of (S)-naproxen O-demethylation by CYP2C9 using activity-phenotyped human liver microsomes and selective cytochrome P450 inhibitors and then developed and validated a novel liquid chromatography mass spectrometry method for simultaneous quantification of (S)naproxen, (S)-O-desmethylnaproxen, and naproxen acyl glucuronide in human urine. The average ratio of (S)-O-desmethylnaproxen to unchanged $(S)$-naproxen in urine was $18.0 \pm 8.0(n=11)$ for the homozygous CYP2C9 Met1 reference group and $10.3 \pm 6.6(n=11)$ for the Leu1 variant carrier group $(P=0.011)$. The effect of $M 1 L$ variation on CYP2C9 function and its potential to alter the pharmacokinetics of drugs metabolized by the enzyme has clinical implications and should be included in a variant screening panel when pharmacogenetic testing in the Alaska Native population is warranted.

\section{SIGNIFICANCE STATEMENT}

The novel CYP2C9 Met1Leu variant in Alaska Native people was recently identified. This study validated (S)-naproxen as a CYP2C9 probe substrate to characterize the in vivo functional activity of the CYP2C9 Leu1 variant. The results of this pharmacogeneticpharmacokinetic study suggest that the CYP2C9 Leu1 variant exhibits loss of enzyme activity. This finding may be important to consider when administering narrow-therapeutic-index medications metabolized by CYP2C9 and also compels further investigation to characterize novel genetic variation in understudied populations.

\section{Introduction}

The CYP2C9 enzyme is responsible for the elimination of approximately $15 \%$ of all medications cleared through a P450-mediated biotransformation pathway (Zanger et al., 2008; Van Booven et al., 2010). CYP2C9 has a broad range of clinical substrates, including anticoagulants, anticonvulsants, angiotensin II blockers, hypoglycemic agents, and nonsteroidal anti-inflammatory drugs. The $C Y P 2 C 9$ gene is

We gratefully acknowledge financial support for this work by National Institutes of Health National Institute of General Medical Sciences [Grant P01-GM116691].

The authors declare no conflicts of interest.

Part of this work was presented in the following doctoral dissertation: Lindsay M. Henderson (2019) Impact of Warfarin Pharmacogene Variation on Drug Metabolism and Pharmacological Response in Alaska Native and American Indian Populations. Doctoral dissertation, University of Washington, Seattle, WA. https://doi.org/10.1124/dmd.120.000301.

S This article has supplemental material available at dmd.aspetjournals.org. highly polymorphic, with coding-region variation $(C Y P 2 C 9 * 2$ and $* 3)$ that confers poor metabolizer phenotype, dramatically influencing the pharmacokinetics and drug response of commonly used narrowtherapeutic-index medications [e.g., $(S)$-warfarin, phenytoin] (Caudle et al., 2014; Flora et al., 2017; Johnson et al., 2017).

Recently, our group identified the novel CYP2C9 Met1Leu (M1L) variant in the Alaska Native (AN) population (Fohner et al., 2015). The substitution of leucine for methionine at the first amino acid position is predicted to markedly slow or stop RNA translation. Indeed, in vitro studies with $M 1 L$ gene-transfected HepG2 cells demonstrated that the CYP2C9 Leu1 variant protein does not accumulate in this liver-derived cell line (McDonald et al., 2020). In the Yup'ik AN population, the M1L variant is found at a higher minor allele frequency $(6.3 \%)$ than the well characterized $C Y P 2 C 9 * 2(0.3 \%)$ and $C Y P 2 C 9 * 3(2.1 \%)$ alleles (Fohner et al., 2015). The historical home of the Yup'ik people is southwestern Alaska, along the Bering Sea, including the relatively remote YukonKuskokwim (YK) Delta. There are 58 communities in the YK Delta

ABBREVIATIONS: AN, Alaska Native; COAG, Clarification of Optimal Anticoagulation through Genetics; EU-PACT, European Pharmacogenetics of Anticoagulant Therapy; HLM, human liver microsome; HPLC, high-performance liquid chromatography; LC/MS, liquid chromatography mass spectrometry; M1L, CYP2C9 Met/Leu; OHSU, Oregon Health \& Science University; P450, cytochrome P450; QC, quality control; rs, reference single nucelotide polymorphism; W, University of Washington; YK, Yukon-Kuskokwim. 
(total population $\sim 23,000$ ), a 75,000-square-mile area, and all are accessed by air, water, or other nonroad system travel. Communities have health clinics staffed by community health aids, and primary care is offered through five subregional health clinics or the regional hub hospital in Bethel, Alaska. This geographic isolation of communities away from primary care providers creates challenges to medical service that may not be experienced in urban areas. For example, pharmacotherapy with narrow-therapeutic-index drugs can be more difficult to manage because of geographical barriers to monitoring drug responses. With specific regard to CYP2C9 substrates, such as warfarin, phenytoin, and tolbutamide, variation in the $C Y P 2 C 9$ gene contributes to interindividual differences in dose requirement (Becker et al., 2008; Caudle et al., 2014; Flora et al., 2017; Johnson et al., 2017). Genetic testing, as a form of precision medicine, has been adopted by many urban medical centers and may have enhanced clinical utility for managing these and other drug therapies in geographically isolated populations. To advance the goals of precision medicine for AN people, it is necessary to fully understand the frequency and function of variation in important pharmacogenes such as $C Y P 2 C$. Moreover, it is critical to investigate previously unknown variants, such as $M 1 L$ and $N 218 I$, that are common in the AN population (Fohner et al., 2015) and are expected to impair CYP2C9 activity.

Characterization of enzyme function in vivo is commonly accomplished with a pharmacokinetic study that involves administration of a probe drug selectively metabolized by the enzyme of interest. Established CYP2C9 probes include the narrow-therapeutic-index drugs warfarin, phenytoin, and tolbutamide, as well as the nonsteroidal antiinflammatory drugs celecoxib and flurbiprofen. However, for a study in the Yup'ik population, selection of a commonly used drug known to be safe and recognizable to potential participants (over the counter) was considered just as important as selectivity for CYP2C9 activity. Thus, we elected to validate and use $(S)$-naproxen as the in vivo enzyme probe. $(S)$-Naproxen undergoes $O$-dealkylation primarily by CYP2C9, with minor involvement from other P450 enzymes (Miners et al., 1996; Tracy et al., 1997). It is well absorbed (Runkel et al., 1972; Davies and Anderson, 1997), highly bound to albumin (Davies and Anderson, 1997), and almost completely eliminated in the urine as naproxen glucuronide $(60 \%$ of the dose), unchanged naproxen $(1 \%)$, and secondary glucuronide and sulfate metabolites of $(S)-O$ desmethylnaproxen (20\%) (Sugawara et al., 1978; Kiang et al., 1989; Vree et al., 1993; Davies and Anderson, 1997). Although not the major pathway of $(S)$-naproxen elimination, a low total urinary $(S)-O$ desmethylnaproxen/ $(S)$-naproxen concentration ratio is indicative of a low CYP2C9 intrinsic formation clearance. This study's objective was to verify the selectivity of the $(S)$-naproxen $O$-dealkylation reaction for CYP2C9 and then determine the catalytic efficiency of the novel $M 1 L$ variant in vivo to inform on its potential to affect the drug disposition and pharmacological response of medications metabolized by CYP2C9.

\section{Materials and Methods}

Setting. Study recruitment was conducted in 10 communities found in the YK Delta of Alaska. Approximately two-thirds of the AN population in Alaska live in rural communities with populations of 50-1000 people, many only accessible by air or water (Norris et al., 2012). Dr. Bert Boyer and Ms. Scarlett Hopkins, formerly at the University of Alaska Fairbanks and now based at Oregon Health \& Science University (OHSU), have ongoing genetic research partnerships with 11 of the 58 rural communities in the YK Delta.

Study Participants. Study participants were selected from a cross-sectional population of Yup'ik men and women over 18 years old, for whom CYP2C9 $M 1 L$ genotype was previously determined and who consented to be contacted for future research investigations. Participants were in good health and not taking nonsteroidal anti-inflammatory agents or other drugs known or suspected of altering CYP2C9 function.

Study Design. The University of Alaska Fairbanks and OHSU institutional review boards and the Yukon-Kuskokwim Health Corporation human studies committee and executive board approved this study. The University of Washington (UW) institutional review board approved the overall research project, as UW was the academic home of the grant funding this research (National Institutes of Health P01-GM1 16691) and its principal investigators. The study is registered at clinicaltrials.gov (NCT04449471).

After written informed consent, participants were asked to fast for 12 hours prior to the start of the pharmacokinetic study and then provided a baseline urine sample. A single $220-\mathrm{mg}$ naproxen sodium caplet [200 mg (S)-naproxen] was administered with a glass of water. Urine was collected for the next 24 hours after the naproxen dose. Because of the instability of naproxen acyl glucuronides in alkaline media, urine $\mathrm{pH}$ was stabilized by adding $13.6 \mathrm{~g}$ monobasic potassium phosphate to each urine collection container before use. At the end of the collection interval, study participants returned the urine collection container to the study site, where the urine volume was measured and recorded. The urine was well mixed, and two 5-ml aliquots were taken from the collection container and stored initially at $-15^{\circ} \mathrm{C}$ in a portable freezer and then at $-80^{\circ} \mathrm{C}$ until analysis.

Genotyping. To identify Met1/Leu1 heterozygotes and Leul/Leu1 homozygotes from the Yup'ik population, the Fluidigm platform was used to perform genotype analysis of DNA extracted from white blood cells, targeting the CYP2C9 exome, as previously described (Fohner et al., 2015). Based on prior gene sequencing work, the following $C Y P 2 C 9$ variants (cDNA position and base change indicated for variants without a reference single nucleotide polymorphism (rs) identification number) were tested: Met1Leu $(1 \mathrm{~A}>\mathrm{T})$, Asn218Ile (653A > $\mathrm{T}), * 2$ (rs1799853), *3 (rs1057910), *8 (rs7900194), *11 (rs28371685), *13 (rs72558187), *14 (rs72558189), and $* 29$ (rs182132442). A total of 1112 individuals from the Yup'ik population were genotyped.

Validation of (S)-Naproxen as a Selective CYP2C9 Probe Substrate. Comprehensive in vitro studies were performed to validate the selectivity and sensitivity of naproxen as a probe for CYP2C 9 activity. Unlabeled $(S)$-naproxen and racemic $O$-desmethylnaproxen- $\mathrm{d}_{3}$ were purchased from Toronto Research Chemicals (ON, Canada). Unlabeled $O$-desmethylnaproxen, furafylline, sulfaphenazole, and NADPH were purchased from Sigma Aldrich (St. Louis, MO). Pooled human liver microsomes (HLMs) were purchased from XenoTech (Kansas City, KS). Individual HLMs were isolated from the University of Washington School of Pharmacy human liver bank, as previously reported (Shirasaka et al., 2016). Individual recombinantly expressed cytochrome P450 Supersome preparations were obtained from Corning Life Sciences (Woburn, MA). All other chemicals were of analytical grade or better and obtained from various commercial vendors.

$(S)$-Naproxen was incubated with pooled HLMs $(0.5 \mathrm{mg} / \mathrm{ml}$ final concentration) in the presence of NADPH ( $1 \mathrm{mM}$ final concentration) in a buffer consisting of $50 \mathrm{mM} \mathrm{KH}_{2} \mathrm{PO}_{4}$ with $1.27 \mathrm{mM}$ EDTA, pH 7.4, at a total volume of $200 \mu \mathrm{l}$. In experiments using selective $\mathrm{P} 450$ isoform inhibitors sulfaphenazole (prepared in methanol, with final concentration below $0.2 \%$ ) and furafylline (prepared in DMSO, with final concentration below $0.1 \%$ ), the final inhibitor concentration was $10 \mu \mathrm{M}$. Microsomal incubations with furafylline underwent a 20 -minute preincubation with the CYP1A2 inhibitor prior to $(S)$-naproxen reaction initiation. Reactions ran for 20 minutes at $37^{\circ} \mathrm{C}$ and were conducted over an $(S)$-naproxen concentration range of 5-1800 $\mu \mathrm{M}$. The microsomal reaction was quenched with the addition of $1 \mathrm{ml}$ ice-cold methanol containing $2 \%$ formic acid. To the quenched samples, $80 \mathrm{ng}$ of $O$-desmethylnaproxen- $\mathrm{d}_{3}$, internal standard, was added. The samples were then centrifuged at $3000 \mathrm{~g}$ for 10 minutes, decanted into glass culture tubes, dried with nitrogen gas, and resuspended in $50 \mu \mathrm{l}$ mobile phase. A volume of $20 \mu \mathrm{l}$ was injected onto the LC/MS.

A P450 Supersome screen was performed by evaluating CYP1A1, CYP1A2, CYP2A6, CYP2B6, CYP2C8, CYP2C9*1, CYP2C9*2, CYP2C9*3, CYP2C19, CYP2D6, CYP2E1, CYP3A4, CYP3A5, and CYP3A7 metabolic activity toward $(S)$-naproxen. For this experiment, $(S)$-naproxen was incubated with 10 pmol of each Supersome preparation in $50 \mathrm{mM} \mathrm{KH}_{2} \mathrm{PO}_{4}$ with $1.27 \mathrm{mM}$ EDTA buffer (except CYP2A6, for which $50 \mathrm{mM}$ Tris buffer was used) in a total volume of $200 \mu \mathrm{l}$. Reactions were initiated with the addition of NADPH (1 mM final concentration) and incubated for 20 minutes at $37^{\circ} \mathrm{C}$ at the (S)-naproxen concentrations of $25 \mu \mathrm{M}$ (below $\mathrm{K}_{\mathrm{m}}$ ) and $1000 \mu \mathrm{M}$ (saturating concentration). The incubation reaction was quenched with $1 \mathrm{ml}$ ice-cold methanol containing 
$2 \%$ formic acid, and $200 \mathrm{ng}$ of $O$-desmethylnaproxen- $\mathrm{d}_{3}$, internal standard, was added. The samples were centrifuged at $3000 \mathrm{~g}$ for 10 minutes, decanted into glass culture tubes, dried with $\mathrm{N}_{2}$ gas, and resuspended in $50 \mu \mathrm{l}$ mobile phase. A volume of $5 \mu \mathrm{l}$ was injected onto the LC/MS. For P450 enzymes that catalyzed (S)-O-desmethylnaproxen formation, additional reactions were carried out for 20 minutes at $37^{\circ} \mathrm{C}$, over the $(S)$-naproxen concentration range of $5-1800 \mu \mathrm{M}$, to determine Michaelis-Menten kinetic parameters. The data were normalized for the absolute amount of recombinant $\mathrm{P} 450$ added to the incubations (i.e., picomole $\mathrm{P} 450)$.

To evaluate the effect of varying CYP1A2 content on naproxen metabolism, single -donor HLMs from the UW human liver bank were selected based on CYP1A2 protein content [determined by liquid chromatography tandem mass spectrometry (LC-MS/MS) analysis]. Five high CYP1A2 expressors and five low CYP1A2 expressors, all with comparable CYP2C9 protein expression, were incubated with $20 \mu \mathrm{M}(S)$-naproxen and NADPH (1 mM final concentration), in the presence or absence of $10 \mu \mathrm{M}$ sulfaphenazole and furafylline, in $50 \mathrm{mM}$ $\mathrm{KH}_{2} \mathrm{PO}_{4}$ with 1.27 mM EDTA, $\mathrm{pH} 7.4$, and in a total volume of $200 \mu \mathrm{l}$. Reactions were carried out as described above with pooled HLM experiments.

Calibration curves for $(S)$-naproxen metabolites were prepared by spiking variable amounts of unlabeled $(S)$ - $O$-desmethylnaproxen into $200 \mu \mathrm{l}$ of potassium phosphate buffer to generate standard mixtures, with final concentrations of $0.2-10 \mu \mathrm{M}$ for HLM incubations, $0.1-5 \mu \mathrm{M}$ for HLM inhibition experiments, and $0.1-30 \mu \mathrm{M}$ for Supersome experiments. Standard solutions prepared in duplicate for each concentration were immediately worked up and analyzed in an identical fashion to that described for the incubation samples above. GraphPad Prism version 8.4.3 (GraphPad Software, San Diego, CA) was used to estimate $\mathrm{K}_{\mathrm{m}}$ and $\mathrm{V}_{\max }$ parameters.

Urine Sample Preparation. For $(S)$-naproxen detection, urine samples $(50 \mu \mathrm{l})$ were prepared by adding $100 \mu \mathrm{l}$ of HPLC-grade water and $100 \mu \mathrm{l}$ of 1 nmol racemic naproxen- $\mathrm{d}_{3}$ [internal standard for $(S)$-naproxen]. For naproxen acyl glucuronide detection, urine samples were diluted 1:20 in blank urine. Then, $50 \mu \mathrm{l}$ of the diluted sample was combined with $100 \mu$ l of HPLC-grade water and $100 \mu \mathrm{l}$ of $1 \mathrm{nmol}$ racemic flurbiprofen acyl glucuronide (internal standard for naproxen acyl glucuronide). For total $(S)$ - $O$-desmethylnaproxen detection, urine was diluted 1:4 in blank urine, and then the diluted urine sample $(50 \mu \mathrm{l})$ was combined with $80 \mu \mathrm{l}$ HPLC-grade water, $20 \mu \mathrm{l} 6 \mathrm{M} \mathrm{HCL}$, and $100 \mu \mathrm{l}$ of internal standard ( $1 \mathrm{nmol}$ racemic $O$-desmethylnaproxen- $\mathrm{d}_{3}$ ) followed by vortexing and incubating at $90^{\circ} \mathrm{C}$ for 60 minutes to facilitate glucuronide and sulfate cleavage via acid hydrolysis. This heated acid hydrolysis approach was adapted from a published protocol for a similarly structured acyl glucuronide (Zgheib et al., 2007), since $O$-desmethylnaproxen glucuronide can hydrolyze back to $O$-desmethylnaproxen or isomerize to glucuronidase-resistant isoglucuronides under alkaline conditions (Davies and Anderson, 1997). All samples were vortexed and centrifuged at $14,000 \mathrm{~g}$ for 5 minutes; then, $50 \mu \mathrm{l}$ of sample supernatant was transferred to autosampler vials, and $2 \mu l$ was injected onto the LC/MS.

Urine Sample Analysis. To evaluate the effect of $M 1 L$ variation on CYP2C9 function, the ratio of urinary $(S)$ - $O$-desmethylnaproxen to unchanged naproxen metabolite to parent was determined from the 24-hour urine collection. Naproxen and metabolite concentrations were accessed by LC/MS using an Agilent 1956B single-quadrupole mass spectrometer coupled with an Agilent 1200 series (Santa Clara, CA) liquid chromatography system. Chromatographic separation was achieved on a Luna C18 $(2 \times 50 \mathrm{~mm} \times 5 \mu \mathrm{m})$ column (Torrence, CA) with a mobile-phase flow rate of $0.3 \mathrm{ml} / \mathrm{min}$. The mobile phase consisted of $10 \mathrm{mM}$ ammonium formate (A, pH 3.5) and methanol (B), and linear gradients were applied with B\% increasing from $40 \%$ to $80 \%$ between 3 and 8 minutes and decreasing to $40 \%$ at 9 minutes. Quantitation was achieved by selected ion monitoring centered on mass-to-charge $(\mathrm{m} / \mathrm{z})$ values of 248.1 for $(S)$-naproxen, 251.1 for racemic naproxen- $\mathrm{d}_{3}, 234.1$ for $(S)$ - $O$-desmethylnaproxen, 237.1 for racemic $O$-desmethylnaproxen- $\mathrm{d}_{3}, 424.1$ for naproxen acyl glucuronide, and 438.1 for racemic flurbiprofen acyl glucuronide. Data acquisition and analysis were performed using the Agilent MassHunter software. Calibration curves were constructed by plotting the peak area ratio of each compound to the respective internal standard against a range of targeted analyte concentrations. We measured the urinary concentration of the major naproxen metabolite, naproxen acyl glucuronide, to ensure comparable dose recovery and urine collection compliance. The intraday variation for quantitation of each analyte did not exceed $2 \%$ for the low-concentration quality control (QC) for $(S)$ - $O$-desmethylnaproxen and did not exceed 6\% for the high-concentration QC. The relative errors of the two QC concentrations tested in three independent experiments were within $5 \%$ and $8 \%$ for the low- and high-concentration QCs, respectively.

Statistical Analysis. We proposed a regression analysis for the statistical analysis plan to test for an additive gene dose effect $(0,1$, or 2 functional alleles) of Leu1. From our power calculations, a sample size of 30, with 10 participants per genotype group, resulted in a power of 0.8 at a 0.05 significance level. Considering possible difficulties in recruiting 10 Leul/Leul homozygotes, we also proposed a priori an alternative statistical analysis plan in which the homozygous variant Leul/Leul group would be combined with the heterozygous variant Met1/Leul group. Under this plan, a sample consisting of 15 reference homozygotes and 15 Leul carriers and homozygotes would achieve the same power at the same level of significance. Recruitment was halted by travel restrictions instituted by OHSU and the Yukon-Kuskokwim Health Corporation because of the coronavirus disease 2019 (COVID-19) pandemic, and our final sample size was 22, with 11 Met1 homozygotes and 11 Leul carriers and homozygotes. Travel is still restricted as of the time of submission of this manuscript. The regression analysis for the comparison between the Met1/Met 1 reference and combined Met1/Leul heterozygotes and Leu1/Leul homozygotes allowed for heteroscedasticity and was completed using RStudio version 1.2.1335 (RStudio, Inc., Boston, MA). With regard to the analysis of the in vitro data, comparisons between recombinant enzymes and single-donor HLMs were assessed using two-tailed unpaired $t$ tests, allowing for heteroscedasticity.

\section{Results}

Study Enrollment Based on M1L Genotype. A total of 1112 Yup'ik adults were genotyped for $C Y P 2 C 9$ variants. After removing duplicate records from repeat visits $(n=193)$ and genotypes with no calls $(n=6)$, a total of 913 genomes were considered for the pharmacokinetic study (Fig. 1). Individuals with one or more copies of $C Y P 2 C 9 * 2, * 3, * 8, * 11, * 13, * 14$, *29, or $N 218 I$ alleles were excluded because of their confounding effects on CYP2C 9 activity. A total of eight Leul/Leul homozygotes were identified from five different communities, with an average age of 36 years. These individuals were all unrelated at the parent-child and sibling level. A total of $85 \mathrm{Met} 1 / \mathrm{Leul}$ heterozygotes, with an average age of 37 years, and 629 in the Met1/Met1 reference (wild type), with an average age of 36 years, were also identified.

Selectivity and Sensitivity of (S)-Naproxen as a Probe for CYP2C9 Enzyme Activity. Comprehensive in vitro studies determined that CYP2C9 is the predominant enzyme metabolizing $(S)$-naproxen to $(S)$-O-desmethylnaproxen. A representative Michaelis-Menten plot of (S)-O-desmethylnaproxen formation in pooled HLMs is shown in Fig. 2, with a mean $\mathrm{K}_{\mathrm{m}}$ of $420 \pm 2 \mu \mathrm{M}$ and $\mathrm{V}_{\max }$ of $0.92 \pm 0.06 \mathrm{nmol} / \mathrm{min}$ per milligram microsomal protein from three repeated experiments. The $\mathrm{P} 450$ Supersome screen showed that $(S)$ - $O$-desmethylnaproxen was formed in incubations containing $25 \mu \mathrm{M}$ naproxen by only three $\mathrm{P} 450$ enzymes-CYP2C9, CYP2C8, and CYP1A2 (Fig. 3). The activity of CYP2C9 was much higher than that of CYP1A2 and CYP2C8. A $25 \mu \mathrm{M}$ concentration is more clinically relevant, considering that $(S)$ naproxen exhibits extensive protein binding (>99\%) and its $\mathrm{C}_{\max }$ is $\sim 250 \mu \mathrm{M}$ after a single 440-mg dose under fasting conditions (Center for Drug Evaluation and Research, 2005). The dominant role of CYP2C9 is less apparent at higher $(S)$-naproxen concentrations, as demonstrated in the incubation with $1000 \mu \mathrm{M}$ of $(S)$-naproxen, for which the contribution of CYP1A2 increased by over 30-fold and small amounts of product were detected in incubations with several other P450 Supersomes (Fig. 3). In a separate experiment, we evaluated the activity of a vector control Supersome preparation compared with CYP2C9. There was no $(S)$ - $O$-desmethylnaproxen product detected in incubations with the vector control at both the 25 and $1000 \mu \mathrm{M}$ substrate concentrations compared with a robust product formation rate produced by CYP2C9. 


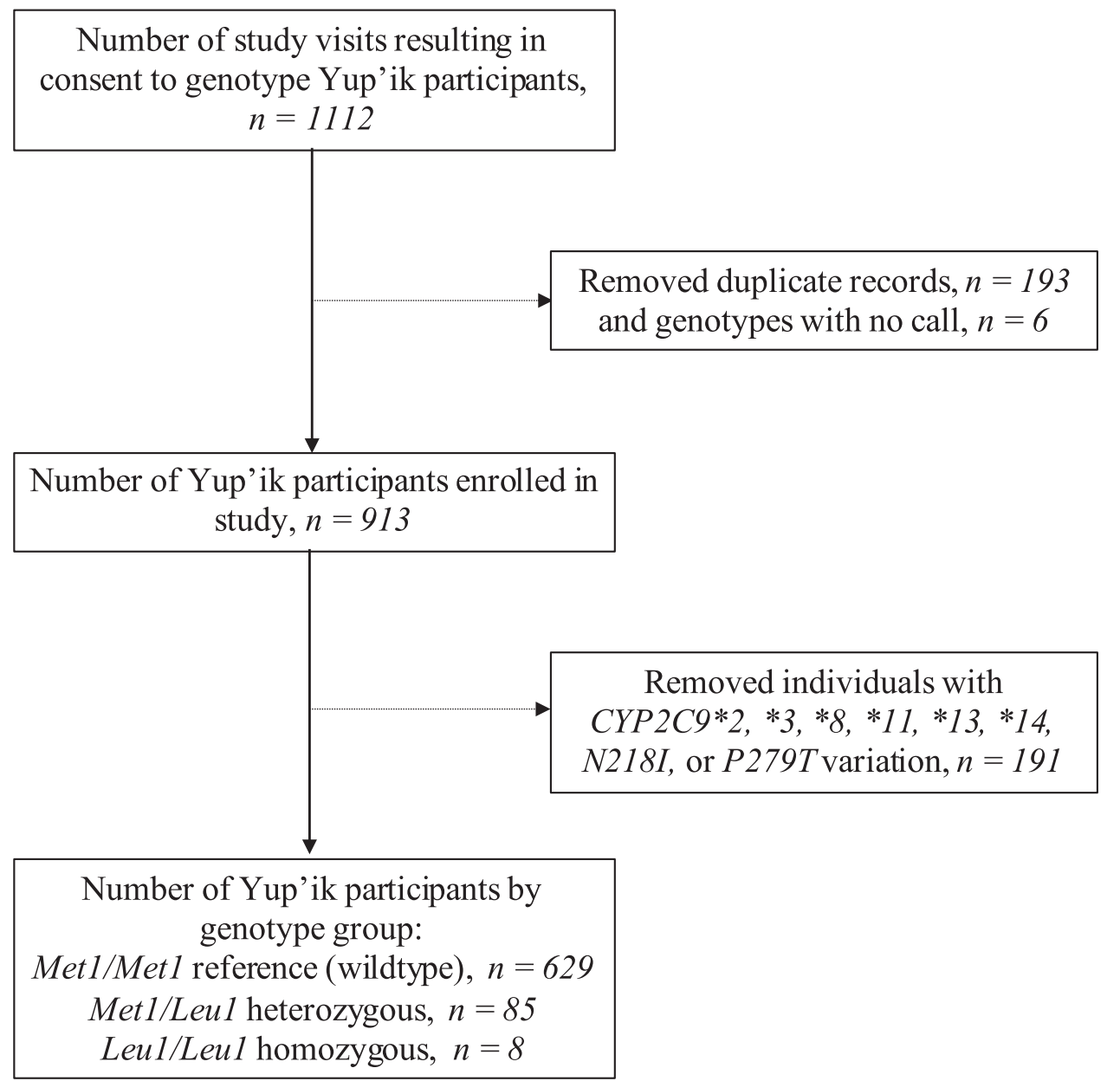

Fig. 1. Study inclusion based on $C Y P 2 C 9 M 1 L$ screening results.

Full kinetic experiments were conducted to assess the $(S)-O$ desmethylnaproxen intrinsic formation clearances of CYP2C9, CYP1A2, and CYP2C8 Supersomes (Fig. 4). The mean $V_{\max }$ values for CYP2C9 and CYP1A2 were 31.7 and $41.7 \mathrm{pmol} / \mathrm{min}$ per picomole
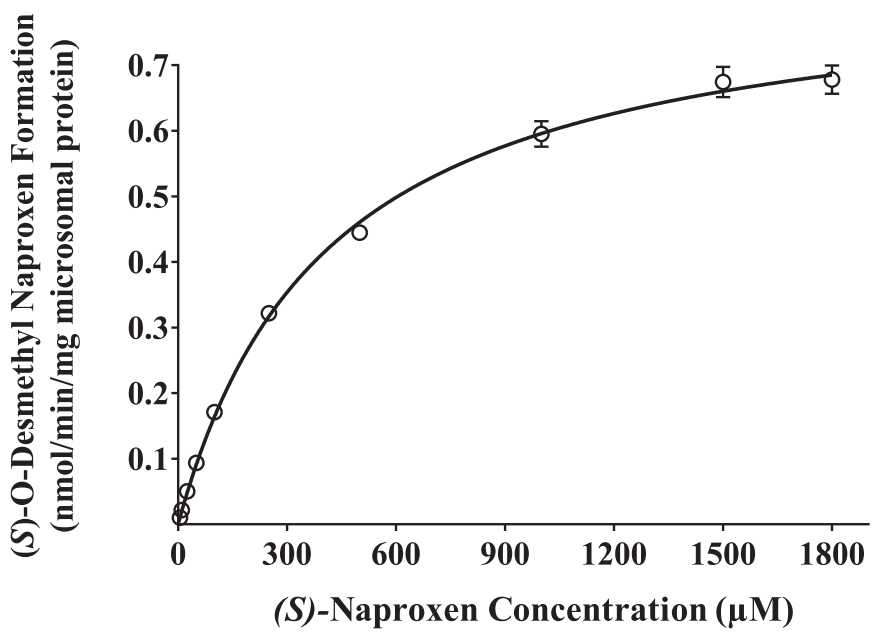

Fig. 2. Representative Michaelis-Menten plot of (S)-O-desmethylnaproxen formation in pooled HLMs. Data are means \pm S.D. Individual data points represent means of technical triplicates at a given $(S)$-naproxen concentration from the same experimental replicate, and the solid line reflects the fit of a single-enzyme Michaelis-Menten model to the data.
P450, respectively, whereas their $\mathrm{K}_{\mathrm{m}}$ values were markedly different: $280 \mu \mathrm{M}$ for CYP2C9 and $1000 \mu \mathrm{M}$ for CYP1A2 $(P=0.005)$ (Table 1$)$. The intrinsic clearance by CYP2C9 was significantly greater than for CYP1A2 $(P=0.008)$. Given liver abundances of 73, 52, and $24 \mathrm{pmol}$ P450 per milligram protein (Rowland-Yeo et al., 2004) for CYP2C9, CYP1A2, and CYP2C8, respectively, the average contribution from each of these enzymes to $(S)$ - $O$-desmethylnaproxen formation was predicted to be $78 \%$ for CYP2C9, $20 \%$ for CYP1A2, and $2 \%$ for CYP2C8

We also estimated the fraction of $(S)$-naproxen metabolized to $(S)-O$ desmethylnaproxen in HLMs by CYP2C9 and CYP1A2 from selective

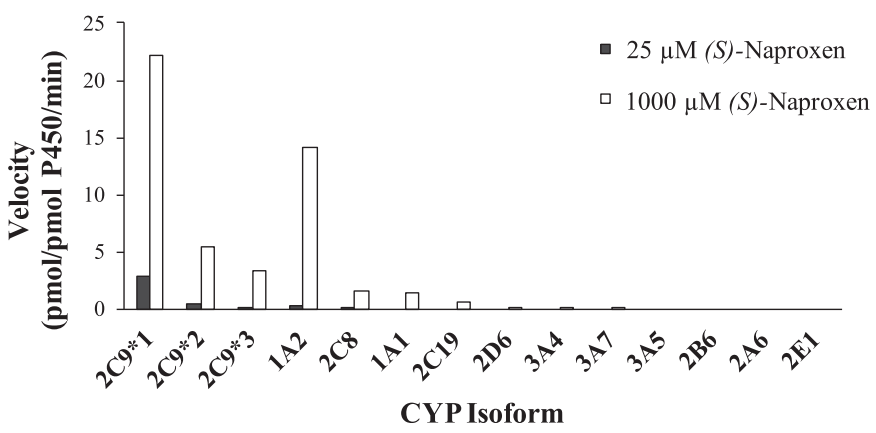

Fig. 3. $\mathrm{P} 450$ Supersome screen at a sub- $\mathrm{K}_{\mathrm{m}}(S)$-naproxen concentration $(25 \mu \mathrm{M}$, closed bars) and a saturating concentration $(1000 \mu \mathrm{M}$, open bars). Data are mean values across two repeated experiments, each with technical triplicates. 


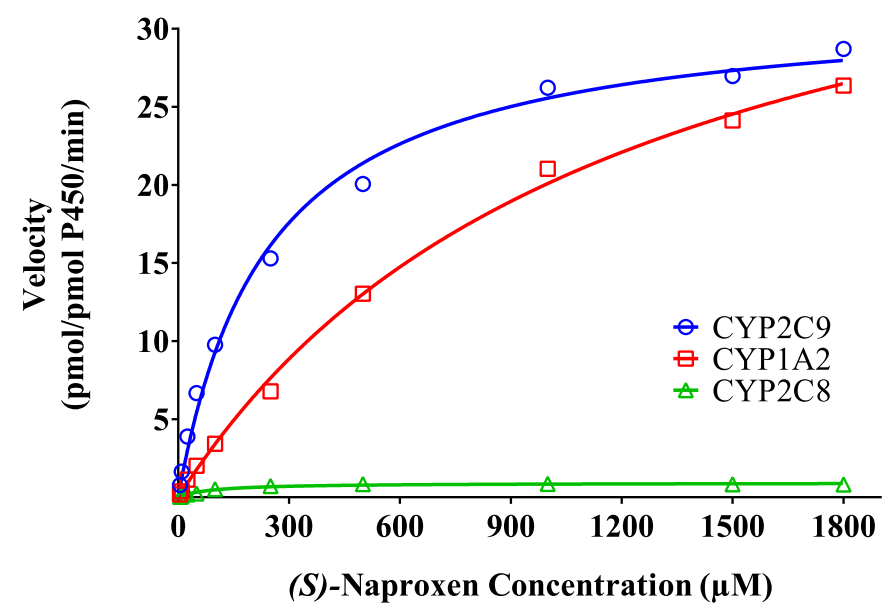

Fig. 4. Michaelis-Menten plot of (S)- $O$-desmethylnaproxen formation by CYP2C9, CYP1A2, and CYP2C8 Supersomes. The displayed results are from a representative experiment. Individual data points represent the means of technical duplicates at a given $(S)$-naproxen concentration, and the solid lines reflect the fit of a singleenzyme Michaelis-Menten model to the data.

enzyme inhibitor experiments conducted with $20 \mu \mathrm{M}(S)$-naproxen, a substrate concentration 5 -fold below the $\mathrm{K}_{\mathrm{m}}$ determined in pooled HLMs. (S)-O-desmethylnaproxen formation was reduced by $76.9 \% \pm$ $1.5 \%$ with $10 \mu \mathrm{M}$ sulfaphenazole, a selective CYP2C9 inhibitor; by $21.5 \% \pm 1.6 \%$ with $10 \mu \mathrm{M}$ furafylline, a selective CYP1A2 inhibitor; and by $95.8 \% \pm 2.1 \%$ with both sulfaphenazole and furafylline (Fig. 5). The solvents for the inhibitors had negligible effects on the percent inhibition (Fig. 5).

The effect of CYP2C9 and CYP1A2 inhibition on (S)-O-desmethylnaproxen formation by HLMs was also assessed in two groups of singledonor HLMs, high CYP1A2 expressors $(n=5)$ with an average CYP1A2 content of $31.2 \pm 10.8 \mathrm{pmol} / \mathrm{mg}$ microsomal protein, and low CYP1A2 expressors $(n=5)$ with an average of $2.8 \pm 2.3 \mathrm{pmol} / \mathrm{mg}$ microsomal protein. CYP2C9 content was $53.2 \pm 13.3$ and $36.6 \pm 6.3$ $\mathrm{pmol} / \mathrm{mg}$ microsomal protein in the high- and low-CYP1A2 groups, respectively. As predicted, the percent inhibited by $10 \mu \mathrm{M}$ furafylline was greater in the high-CYP1A2 group $(39.7 \% \pm 7.0 \% \mathrm{pmol} / \mathrm{mg}$ microsomal protein) compared with the low-CYP1A2 group $(23.6 \% \pm$ $7.6 \% \mathrm{pmol} / \mathrm{mg}$ microsomal protein) $(P=0.008)$, whereas the percent inhibited by $10 \mu \mathrm{M}$ sulfaphenazole was greater in the low-CYP1A2 group $(85.2 \% \pm 11.8 \% \mathrm{pmol} / \mathrm{mg}$ microsomal protein) compared with the high-CYP1A2 group $(65.5 \% \pm 4.1 \% \mathrm{pmol} / \mathrm{mg}$ microsomal protein $)$ $(P=0.017)$ (Table 2). Although the CYP1A2 content was 11-fold greater in the high-CYP1A2 group compared with the low-CYP1A2 group, the percent inhibited by furafylline was only 1.7 -fold greater. Thus, CYP1A2 contribution to $(S)$ - $O$-desmethylnaproxen formation in HLMs was always minor in comparison with the CYP2C9 contribution.

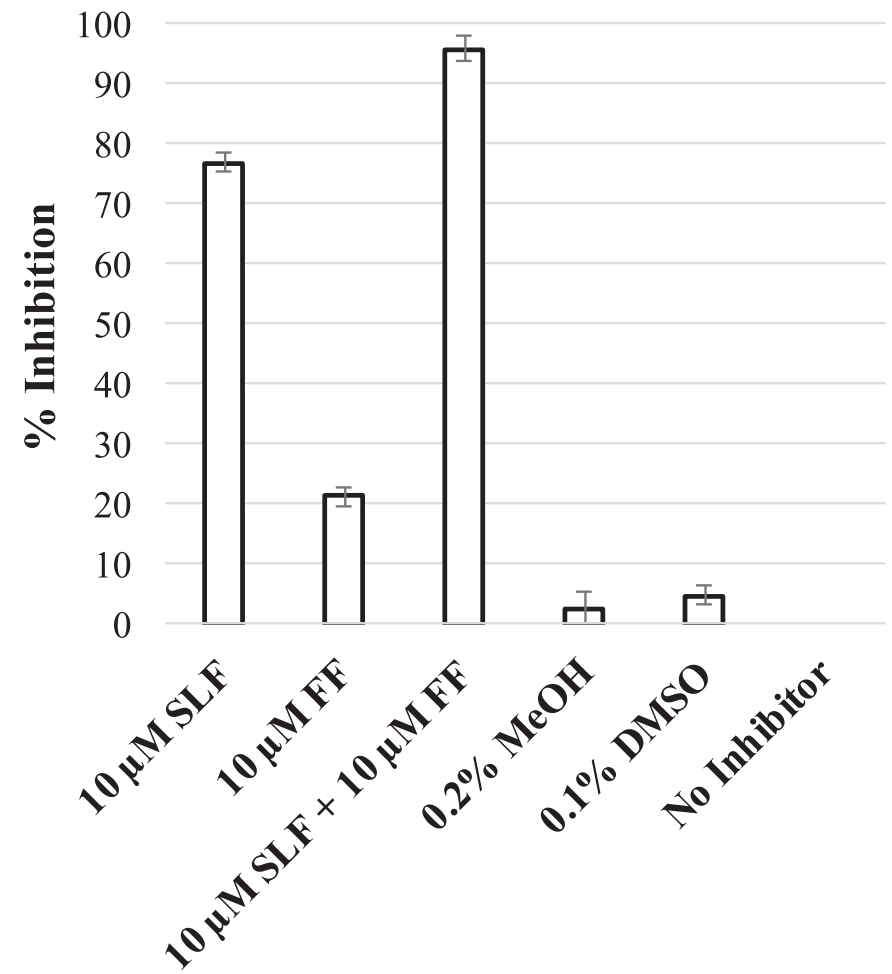

Fig. 5. Effects of selective CYP2C9 and CYP1A2 inhibitors on $(S)$ - $O$-desmethylnaproxen formation in pooled HLMs. Sulfaphenazole (SLF) was dissolved in methanol $(\mathrm{MeOH}),<0.2 \%$ final concentration, and furafylline (FF) was dissolved in DMSO, $<0.1 \%$ final concentration. $(S)$-Naproxen substrate concentration was 20 $\mu \mathrm{M}$. Percent inhibition was calculated relative to the control containing no inhibitor. Data are means \pm S.D. across three repeated experiments, with technical triplicates.

Impact of $M 1 L$ on Urinary Metabolite-to-Parent Ratio. The mean ratio of $(S)$ - $O$-desmethylnaproxen to $(S)$-naproxen was greater for the homozygous reference group $(18.0 \pm 8.0, n=11)$ compared with the $M 1 L$ variant group $(10.3 \pm 6.6, n=11)$, which includes eight Met1/Leu1 heterozygotes and three Leul/Leul homozygotes (Fig. 6). Pairwise comparison (allowing for heteroscedasticity) was significant $(P=0.011)$, indicating reduced activity for the Leul variant. The mean metabolite-to-parent ratios for heterozygotes and Leul/Leul homozygotes were $9.7 \pm 5.6$ and $12.1 \pm 10.1$, respectively (Supplemental Fig. 1). One of the three Leul/Leul homozygote participants reported using tobacco products, which may have induced their CYP1A2 activity and skewed that result and mean for a small sample size. There was no evidence of significant metabolic shifting toward the parent glucuronide elimination pathway in Leul carriers, as the mean urinary metabolite-toparent ratio for the conjugate in carriers of the Leul allele $(32.3 \pm 12.9)$ was similar to that of the reference group $(34.5 \pm 9.4)$.

TABLE 1

Kinetic parameters for $O$-desmethyl $(S)$-naproxen formation by CYP2C9, CYP1A2, and CYP2C8 Supersomes

Reported parameters are the means \pm S.D. across three repeated experiments for CYP2C9 and CYP1A2 Supersomes (duplicate experiments for CYP2C8), each with technical duplicates. Comparisons between CYP2C9 and CYP1A2 were assessed using two-tailed unpaired t tests allowing for heteroscedasticity. The asterisks denote statistical significance when compared with the same kinetic parameter in CYP2C9 Supersomes.

\begin{tabular}{lccc}
\hline P450 & $\mathrm{K}_{\mathrm{m}} \pm$ S.D. & $\mathrm{V}_{\max } \pm$ S.D. & $\mathrm{CL}_{\text {int }} \pm$ S.D. \\
\hline & $\mu M$ & pmol/min per picomole P450 & $\mu l /$ min per picomole P450 \\
CYP2C9 & $280 \pm 8.9$ & $31.7 \pm 2.5$ & $0.11 \pm 0.10$ \\
CYP1A2 & $1000 \pm 97^{*}$ & $41.7 \pm 2.4^{*}$ & $0.04 \pm 0.003 *$ \\
CYP2C8 & 90 & 0.90 & 0.01 \\
\hline
\end{tabular}

${ }^{*} \mathrm{P}<0.01$. 
TABLE 2

Inhibition of $(S)$ - $O$-desmethylnaproxen formation in single-donor HLMs by sulfaphenazole and furafylline

Data are means \pm S.D. across three repeated experiments. Comparisons between low $(n=5)$ and high $(n=5)$ CYP1A2 expressors were assessed using two-tailed unpaired $t$ tests allowing for heteroscedasticity. The asterisks denote statistical significance when compared with percent inhibition in low CYP1A2 expressors.

\begin{tabular}{|c|c|c|c|c|}
\hline $\begin{array}{l}\text { CYP1A2 } \\
\text { Expression }\end{array}$ & Average CYP1A2 \pm S.D. & Average CYP2C9 \pm S.D. & Inhibition by Furafylline \pm S.D. & Inhibition by Sulfaphenazole \pm S.D. \\
\hline & pmol/mg microsomal protein & pmol/mg microsomal protein & $\%$ & $\%$ \\
\hline Low & $2.8 \pm 2.3$ & $36.6 \pm 6.3$ & $23.6 \pm 7.6$ & $85.2 \pm 11.8$ \\
\hline High & $31.2 \pm 10.8$ & $53.2 \pm 13.3$ & $39.7 \pm 7.0 * *$ & $65.5 \pm 4.1^{*}$ \\
\hline
\end{tabular}

${ }^{*} P<0.05 ; * * P<0.01$.

\section{Discussion}

Alaska Native people are under-represented in genetic research but have unique pharmacogene variation that may critically impact their response to drug therapy. This is the first study to characterize prospectively the in vivo functional effect of the novel, relatively common CYP2C9 MIL single nucleotide polymorphism identified in Yup'ik and other AN people. The results suggest that a change in the start codon conferred complete loss of function with no protein synthesis. Given the mean contributions of CYP2C9 (80\%) and CYP1A2 (20\%) to $(S)$-O-desmethylnaproxen formation in HLMs, it was predicted that a Leul variant group (composed of three Leul/Leul homozygotes and eight heterozygotes) would have a $51 \%$ reduction in urinary ratio of $(S)$ - $O$-desmethylnaproxen to unchanged naproxen compared with the reference group. The observed $43 \%$ reduction in the Leul variant group is in good agreement with this prediction. A loss of enzyme activity with the Leul variant has clinical implications, particularly for drugs with a low narrow therapeutic index, such as warfarin, phenytoin, and tolbutamide, for which carriers of the variant would be more likely to experience an exaggerated drug response. In addition, failure to include this variant in a pharmacogenetic test panel, if implemented to

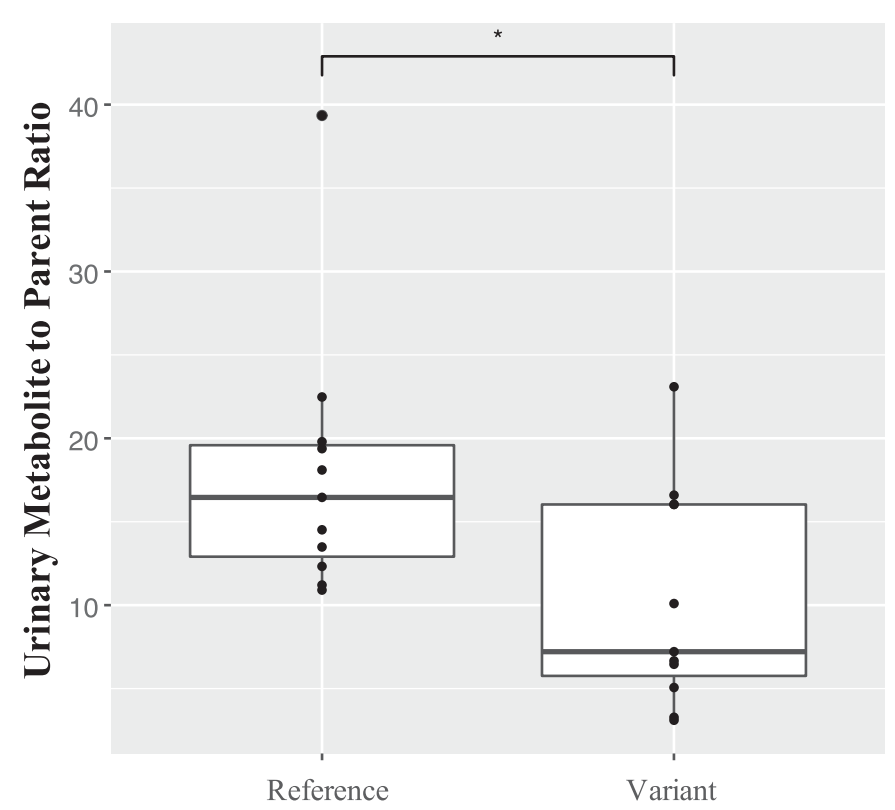

\section{CYP2C9 M1L Genotype Group}

Fig. 6. Urinary metabolite-to-parent ratio of $(S)-O$-desmethylnaproxen to unchanged $(S)$-naproxen by $M 1 L$ genotype. The regression analysis for the comparison between the CYP2C9 Met1/Metl reference group $(n=11)$ and Leul variant carrier group (combined Met1/Leul heterozygotes and Leul/Leul homozygotes) $(n=11)$ allowed for heteroscedasticity; $* P<0.05$. guide drug dose selection, could result in phenotypic misclassification in the Yup'ik population.

The $M 1 L$ variant is a novel $C Y P 2 C 9$ impaired function variant found in the Yup'ik population (and at a lower frequency in other AN groups) (Fohner et al., 2015), but it is not the only example of loss of the translation start codon conferring poor metabolizer status in the P450 2C subfamily. CYP2C19*4 (rs28399504) is a loss-of-function allele that results from a substitution of methionine to valine at the first amino acid position (Ferguson et al., 1998). However, based on data from 1000 genomes, the $C Y P 2 C 19 * 4$ variant is only found at low frequencies across world populations: $0.8 \%$ in a Mexican population (California), $0.5 \%$ in a Han Chinese population (Beijing, China), and the allele was not detected in Europeans (Utah residents with northern and western European ancestry) or in African Americans (southwestern United States) (Auton et al., 2015). By contrast, $M 1 L$ is present at a relatively high minor allele frequency of $6.3 \%$ in the Yup'ik population and thus can contribute to variability in the clearance of CYP2C9 substrates and the associated pharmacological responses.

To characterize the catalytic efficiency of the $M 1 L$ variant, this study first had to establish the use of $(S)$-naproxen as an over-thecounter probe substrate to assess CYP2C9 enzyme activity. Earlier studies characterizing the in vitro metabolism of $(S)$-naproxen downplayed its utility as a probe substrate because of involvement of CYP1A2 (Miners et al., 1996; Rodrigues, 2005) and because an in vivo study in a Korean population did not observe a difference in the mean plasma concentration-time profile of $(S)$-naproxen in $C Y P 2 C 9 * 1 / * 3$ compared with CYP2C9 reference individuals (Bae et al., 2009). However, lack of change in $(S)$-naproxen concentration alone does not provide evidence for the absence of a pharmacogenetic-pharmacokinetic relationship between $C Y P 2 C 9$ genotype and naproxen metabolism because $(S)$-naproxen is eliminated primarily by direct glucuronidation (60\% of the dose) (Vree et al., 1993). Only $20 \%$ of the dose is eliminated as $(S)$ - $O$-desmethylnaproxen and its secondary glucuronide and sulfate metabolites (Sugawara et al., 1978; Kiang et al., 1989; Vree et al., 1993; Davies and Anderson, 1997). Therefore, to detect the effect of CYP2C9 variation on $(S)$-naproxen, it is necessary to consider both the unchanged $(S)$-naproxen as well as its metabolites that are cleared through a CYP2C9-mediated pathway, as was done in the current study. Furthermore, the in vitro experiments conducted here demonstrate that, at physiologically relevant concentrations, CYP2C9 is the major enzyme responsible for naproxen $O$-dealkylation and that CYP1A2 only plays a minor role. Moreover, the results of inhibitor experiments conducted in single-donor HLMs demonstrate that the overall contribution of CYP1A2 to $(S)$ - $O$-desmethylnaproxen formation does not increase substantially with increasing CYP1A2 protein abundance (Table 2). Thus, elevated CYP1A2 expression and activity, due to genotype (Thorn et al., 2012) or xenobiotic exposure (Zevin and Benowitz, 1999; Dobrinas et al., 2011), is not expected to significantly impact CYP2C9's predominant role in the $O$-demethylation of $(S)$-naproxen in vivo. Although flurbiprofen could be considered a more CYP2C-selective 
in vivo probe than $(S)$-naproxen, it was not deemed superior for this study because of concerns with using a drug available only by prescription in communities without local physician oversight and a recommendation by our community advisors that we use a probe drug familiar to the population (available over the counter) to enhance recruitment.

The identification of a novel $C Y P 2 C 9$ variant that impairs enzyme function and is unique to a population under-represented in biomedical, and especially genetic, research (Popejoy and Fullerton, 2016) illustrates the importance of population-specific pharmacogenetic studies to guide medication therapy. A pharmacogenetic algorithm that is based on polymorphisms from a specific subset of the global population may not be as clinically beneficial for populations in which the frequency of variant alleles is markedly different, or if enzyme activity is determined by uncharacterized genetic variation. This was demonstrated by the conflicting results published by two randomized clinical trials, the European Pharmacogenetics of Anticoagulant Therapy (EU-PACT) (Pirmohamed et al., 2013) and the Clarification of Optimal Anticoagulation through Genetics (COAG) (Kimmel et al., 2013) trials. The EU-PACT trial showed a benefit for genotype-guided warfarin dosing over standard clinical care, but the COAG trial did not find a significant difference between the two groups (Kimmel et al., 2013; Pirmohamed et al., 2013). Variation in the ethnicities and genetics of the sample populations likely contributed to the different results (Scott and Lubitz, 2014). Although the EU-PACT participants were primarily European, the COAG study population included 27\% African Americans, who have lower frequencies of $C Y P 2 C 9 * 2$ and $* 3$ (the only $C Y P 2 C 9$ variant alleles considered in the pharmacogenetic algorithm) but higher frequencies of other reduced-function $C Y P 2 C 9$ variants (e.g., *5, *6, $* 8$, and $* 11$ ) (Limdi et al., 2015). Similarly, current pharmacogenetic warfarin dose algorithms would likely not optimize warfarin dosing for the Yup'ik population, in which the $C Y P 2 C 9 * 2$ and $* 3$ frequencies are low and novel reduced or loss-of-function variants, such as $M 1 L$, are present. Although the loss of CYP2C9 enzyme activity predicted for the $M 1 L$ variant may be greater than with $C Y P 2 C 9 * 3(80 \%$ decrease in enzymatic activity) (Takanashi et al., 2000), dosing recommendations for the $C Y P 2 C 9 * 3$ variant can provide some clinical guidance. The Gage pharmacogenetic algorithm recommends a warfarin dose reduction of $33 \%$ per $C Y P 2 C 9 * 3$ allele (Gage et al., 2008). The most conservative guidance would be to switch $M 1 L$ homozygotes to alternative direct oral anticoagulant therapy. Future work should aim to establish the effect of the $M 1 L$ variant on warfarin dose requirement, for example through a prospective study to inform pharmacogenetically guided warfarin dosing algorithms, controlling for other genetic determinants, notably vitamin $\mathrm{K}$ epoxide oxidase reductase complex subunit 1 genotype (Rieder et al., 2005). The Yup'ik population may benefit from the consideration and inclusion of population-specific genetic variation in clinical decisions surrounding personalized medication therapy. Clearly, an understanding of genetic variation in under-represented minority populations is essential if pharmacogenetic testing is to reach its optimal clinical utility in patients of all ethnicities.

\section{Acknowledgments}

The authors would like to thank our Yup'ik study participants, the Yup'ik Community Planning Group, and the Vice President of Quality at the YukonKuskokwim Health Corporation, Dr. Joseph Klejka, for helpful discussions in the design of this research. Lastly, the authors would like to thank Barbara Kavanaugh for her management of human subjects study approvals.

\section{Authorship Contributions}

Participated in research design: Henderson, Hopkins, Boyer, Thornton, Rettie, Thummel.
Conducted experiments: Henderson, Hopkins, Boyer.

Performed data analysis: Henderson, Thornton.

Wrote or contributed to the writing of the manuscript: Henderson, Hopkins, Boyer, Thornton, Rettie, Thummel.

\section{References}

Auton A, Brooks LD, Durbin RM, Garrison EP, Kang HM, Korbel JO, Marchini JL, McCarthy S, McVean GA, and Abecasis GR; 1000 Genomes Project Consortium (2015) A global reference for human genetic variation. Nature 526:68-74.

Bae JW, Kim JH, Choi CI, Kim MJ, Kim HJ, Byun SA, Chang YS, Jang CG, Park YS, and Lee SY (2009) Effect of CYP2C $9 * 3$ allele on the pharmacokinetics of naproxen in Korean subjects. Arch Pharm Res 32:269-273.

Becker ML, Visser LE, Trienekens PH, Hofman A, van Schaik RH, and Stricker BH (2008) Cytochrome P450 2C9 $* 2$ and $* 3$ polymorphisms and the dose and effect of sulfonylurea in type II diabetes mellitus. Clin Pharmacol Ther 83:288-292.

Caudle KE, Rettie AE, Whirl-Carrillo M, Smith LH, Mintzer S, Lee MT, Klein TE, and Callaghan JT; Clinical Pharmacogenetics Implementation Consortium (2014) Clinical pharmacogenetics implementation consortium guidelines for CYP2C9 and HLA-B genotypes and phenytoin dosing. Clin Pharmacol Ther 96:542-548.

Center for Drug Evaluation and Research (2005) Naproxen sodium (220 mg), Clinical Pharmacology and Biopharmaceutics Review(s), New Drug Application 21-920.

Davies NM and Anderson KE (1997) Clinical pharmacokinetics of naproxen. Clin Pharmacokinet 32:268-293.

Dobrinas M, Cornuz J, Oneda B, Kohler Serra M, Puhl M, and Eap CB (2011) Impact of smoking, smoking cessation, and genetic polymorphisms on CYP1A2 activity and inducibility. Clin Pharmacol Ther 90:117-125.

Ferguson RJ, De Morais SM, Benhamou S, Bouchardy C, Blaisdell J, Ibeanu G, Wilkinson GR, Sarich TC, Wright JM, Dayer P, et al. (1998) A new genetic defect in human CYP2C19: mutation of the initiation codon is responsible for poor metabolism of S-mephenytoin. J Pharmacol Exp Ther 284:356-361.

Flora DR, Rettie AE, Brundage RC, and Tracy TS (2017) CYP2C9 genotype-dependent warfarin pharmacokinetics: impact of CYP2C9 genotype on R- and S-warfarin and their oxidative metabolites. J Clin Pharmacol 57:382-393.

Fohner AE, Robinson R, Yracheta J, Dillard DA, Schilling B, Khan B, Hopkins S, Boyer B, Black $\mathrm{J}$, Wiener H, et al. (2015) Variation in genes controlling warfarin disposition and response in American Indian and Alaska Native people: CYP2C9, VKORC1, CYP4F2, CYP4F11, GGCX. Pharmacogenet Genomics 25:343-353.

Gage BF, Eby C, Johnson JA, Deych E, Rieder MJ, Ridker PM, Milligan PE, Grice G, Lenzini P, Rettie AE, et al. (2008) Use of pharmacogenetic and clinical factors to predict the therapeutic dose of warfarin. Clin Pharmacol Ther 84:326-331.

Johnson JA, Caudle KE, Gong L, Whirl-Carrillo M, Stein CM, Scott SA, Lee MT, Gage BF, Kimmel SE, Perera MA, et al. (2017) Clinical Pharmacogenetics Implementation Consortium (CPIC) guideline for pharmacogenetics-guided warfarin dosing: 2017 update. Clin Pharmacol Ther 102:397-404.

Kiang CH, Lee C, and Kushinsky S (1989) Isolation and identification of 6-desmethylnaproxen sulfate as a new metabolite of naproxen in human plasma. Drug Metab Dispos 17:43-48.

Kimmel SE, French B, Kasner SE, Johnson JA, Anderson JL, Gage BF, Rosenberg YD, Eby CS, Madigan RA, McBane RB, et al.; COAG Investigators (2013) A pharmacogenetic versus a clinical algorithm for warfarin dosing. N Engl J Med 369:2283-2293.

Limdi NA, Brown TM, Yan Q, Thigpen JL, Shendre A, Liu N, Hill CE, Arnett DK, and Beasley TM (2015) Race influences warfarin dose changes associated with genetic factors. Blood 126: 539-545.

McDonald MG, Henderson LM, Ray S, Yeung CK, Johnson AL, Kowalski JP, Hanenberg H, Wiek C, Thummel KE, and Rettie AE (2020) Heterologous expression and functional characterization of novel CYP2C9 variants identified in the Alaska native people. J Pharmacol Exp Ther 374:233-240.

Miners JO, Coulter S, Tukey RH, Veronese ME, and Birkett DJ (1996) Cytochromes P450, 1A2, and $2 \mathrm{C} 9$ are responsible for the human hepatic O-demethylation of R- and S-naproxen. Biochem Pharmacol 51:1003-1008.

Norris T, Vines PL, and Hoeffel EM (2012) The American Indian and Alaska Native Population: 2010, in 2010 Census Briefs, U.S. Department of Commerce Economics and Statistics Administration, U.S. Census Bureau, Washington, DC.

Pirmohamed M, Burnside G, Eriksson N, Jorgensen AL, Toh CH, Nicholson T, Kesteven P, Christersson C, Wahlström B, Stafberg C, et al.; EU-PACT Group (2013) A randomized trial of genotype-guided dosing of warfarin. N Engl J Med 369:2294-2303.

Popejoy AB and Fullerton SM (2016) Genomics is failing on diversity. Nature 538:161-164. Rieder MJ, Reiner AP, Gage BF, Nickerson DA, Eby CS, McLeod HL, Blough DK, Thummel KE, Veenstra DL, and Rettie AE (2005) Effect of VKORC1 haplotypes on transcriptional regulation and warfarin dose. $N$ Engl J Med 352:2285-2293.

Rodrigues AD (2005) Impact of CYP2C9 genotype on pharmacokinetics: are all cyclooxygenase inhibitors the same? Drug Metab Dispos 33:1567-1575.

Rowland-Yeo K, Rostami-Hodjegan A, and Tucker G (2004) Abundance of cytochromes P450 in human liver: a meta-analysis. Br J Clin Pharmacol 57:687-688.

Runkel RA, Kraft KS, Boost G, Sevelius H, Forchielli E, Hill R, Magoun R, Szakacs JB, and Segre E (1972) Naproxen oral absorption characteristics. Chem Pharm Bull (Tokyo) 20: $1457-1466$.

Scott SA and Lubitz SA (2014) Warfarin pharmacogenetic trials: is there a future for pharmacogenetic-guided dosing? Pharmacogenomics 15:719-722.

Shirasaka Y, Chaudhry AS, McDonald M, Prasad B, Wong T, Calamia JC, Fohner A, Thornton TA, Isoherranen N, Unadkat JD, et al. (2016) Interindividual variability of CYP2C19-catalyzed drug metabolism due to differences in gene diplotypes and cytochrome $\mathrm{P} 450$ oxidoreductase content. Pharmacogenomics $J$ 16:375-387.

Sugawara Y, Fujihara M, Miura Y, Hayashida K, and Takahashi T (1978) Studies on the fate of naproxen. II. Metabolic fate in various animals and man. Chem Pharm Bull (Tokyo) 26: $3312-3321$.

Takanashi K, Tainaka H, Kobayashi K, Yasumori T, Hosakawa M, and Chiba K (2000) CYP2C9 Ile359 and Leu359 variants: enzyme kinetic study with seven substrates. Pharmacogenetics 10 95-104. 
Thorn CF, Aklillu E, Klein TE, and Altman RB (2012) PharmGKB summary: very important pharmacogene information for CYP1A2. Pharmacogenet Genomics 22:73-77.

Tracy TS, Marra C, Wrighton SA, Gonzalez FJ, and Korzekwa KR (1997) Involvement of multiple cytochrome P450 isoforms in naproxen O-demethylation. Eur J Clin Pharmacol 52:293-298. Van Booven D, Marsh S, McLeod H, Carrillo MW, Sangkuhl K, Klein TE, and Altman RB (2010) Cytochrome P450 2C9-CYP2C9. Pharmacogenet Genomics 20:277-281.

Vree TB, van den Biggelaar-Martea M, Verwey-van Wissen CP, Vree JB, and Guelen PJ (1993) Pharmacokinetics of naproxen, its metabolite O-desmethylnaproxen, and their acyl glucuronides in humans. Biopharm Drug Dispos 14:491-502.

Zanger UM, Turpeinen M, Klein K, and Schwab M (2008) Functional pharmacogenetics/genomics of human cytochromes P450 involved in drug biotransformation. Anal Bioanal Chem 392 1093-1108.
Zevin S and Benowitz NL (1999) Drug interactions with tobacco smoking. An update. Clin Pharmacokinet 36:425-438.

Zgheib NK, Frye RF, Tracy TS, Romkes M, and Branch RA (2007) Evaluation of flurbiprofen urinary ratios as in vivo indices for CYP2C9 activity. Br J Clin Pharmacol 63: $477-487$.

Address correspondence to: Dr. Kenneth E. Thummel, Department of Pharmaceutics, University of Washington, Box 357610, 1959 NE Pacific, Seattle, WA 98195. E-mail: thummel@uw.edu 